opportunities of treating superficial wounds, to give a trial to Simpson's acupressure, as I feel assured that it is not only the most simple and expeditious, but the most effectual method of arresting bleeding, and of putting wounds into the best position for healing that we have. For wounds of the scalp, or where the skin is tough, a spear-pointed glover's needle is better than the pin with the glass head, recommended to be employed in major operations: for unless the pin is retained for twenty-four or thirty hours, the forceps will be required to withdraw it, and the glass head, instead of an improvement, would be found a disadvantage. On the removal of the needle the thread is to be left to form a scab, and if there is a probability of suppuration ensuing in the wound, the thread may be wet repeatedly with a mixture of carbolic acid and glycerine, as practiced in the Infirmary by Professor Lister, in the conversion of compound fractures into simple ones by the exclusion of the air, by the prevention of the formation of pus, and by the promotion of union of the lips of the wound by first intention.

Transverse Presentation. By Charles David Doig, M.D., M.R.C.S. Edin., Canada West.

THIs position of the mature fœetus in utero is, for sufficient reasons, considered one of the causes of protracted and difficult labour, and a source of danger to the mother. It can, however, be discerned by the hand of the intelligent obstetrician with as much facility as either of the other two classes of presentations, viz., Breech and Cephalic, and, when the proper treatment is timeously practised, much suffering and danger are in many cases obviated.

The following are some inductions taken from cases which I have witnessed, and which are narrated at length in the Medical Times and Gazette.

1. Transverse presentation of the foetus in utero is oftentimes, though not invariably, associated with death of the fœetus.

2. If the fotus be mature, and the maternal pelvis of common size, the labour is lingering, and delivery is not accomplished without interference.

3. If the maternal pelvis be of common size, and the fotus not developed - in other words, a good-sized pelvis and a smallsized fotus-delivery can be accomplished by the unaided 
efforts of the uterus; but even in these circumstances the labour is tedious.

4. Rupture of the uterus is a structural lesion found on dissection to attend labours, and, in the instance of transverse presentation, is at once reasonably attributed to violent and long-continued uterine exertion.

5. Rupture of the uterus is indicated by sudden sinking.

6. The treatment commonly practised by obstetricians, viz., introducing the hand into the uterine cavity at an early period, if possible before the membranes have burst and the fluid drained away, seizing the lower extremities, and at once extracting, is efficacious, and safer than delay. In favourable cases both mother and child are thus extricated from peril.

7. After the membranes have ruptured, and the fluid has drained off, podalic version, as the operative procedure is technically called, is very difficult of performance: in some cases absolutely impossible.

8. Anesthesia by administration of chloroform is an invaluable aid in the performance of the operation, rendering the mother inert, and subduing, or rather preventing uterine spasm.

An occurrence has recently come under my notice to substantiate these inferences, and to suggest that,

9, When delivery cannot be accomplished by turning, dismemberment of the foetus can be performed, and the mother thereby be restored to safety and health.

$H$. H., aged 40, large, plethoric, fleshy woman, has been married seventeen years, has had four living children, and, last year, one still-born. Had two abortions, one at six weeks, the other at nine weeks.

On Saturday, 16th June, 1866, patient was seized with pains of a doubtful character in the abdomen, regular labour pains supervening all the Monday following. The membranes ruptured and the fluid drained away on Wednesday morning.

These particulars I ascertained on visiting the patient this morning (22d inst), between three and four, her husband having called at my residence about one in the morning and solicited my attendance.

On examination, the left superior extremity is in the vagina, the shoulder can be felt at the mouth of the uterus, and the uterus is high up above the brim of the pelvis, closely and strongly contracted round the foetus. The pains are neither exceedingly strong nor frequent.

I endeavoured three times to introduce my hand within the cavity of the uterus to get hold of an inferior extremity and change the position of the child, but desisted, baffled by the 
strong and prolonged uterine efforts thereby aroused. In the intervals the pains were much feebler, and patient slept a little time. I therefore determined to change the treatment, and substitute embryotomy for version.

After the patient had had a short respite (12 a.m.) I amputated the left superior extremity from the thorax, removing the scapula and clavicle; then amputated the right one; subsequently divided the spinal column in the region of the shoulders, and extracted, first the trunk and lower extremities, afterwards the head and neck. The after-birth separated along with the head and neck.

The child was a male, well formed, and of good size, but somewhat putrified.

The instruments used in the course of the operation were an iron rod curved at both ends, a knife sharp only at the extremity, and a towel to obviate slipperyness. Pulse 84 in the morning; rose to 104 in the evening.

23d. Had a good night's rest. Pulse 96. Altogether she is in a favourable condition.

30th. Has had no unfavourable symptom.

Remarks.-No chloroform was used in the treatment of this patient. She had been in labour four days (forty-eight hours before rupture of the membranes, and forty-eight hours after rupture of the membranes) before interference was attempted During the eight hours of my personal observation not the slightest advance was made. The foetus had been dead about three weeks; at that time patient had rigors and unpleasant feelings. The greatest difficulty experienced in the operation arose from the uterus being high up in the abdomen. Patient had, previous to my coming, taken castor oil, which had operated freely. She had, moreover, micturated.

\section{CLINICAL RECORD.}

Notes of Clinical Cases of Eye Disease. By D. Argyll Robertson, M.D., F.R.C.S.E, Lecturer on Diseases of the Eye, Edinburgh.

\section{I.-Case of Staphyloma posticum with Hypermetropia.}

Mrs. G., aged 62, consulted me on the 25th of May, 1866, on account of dimness of sight in her left eye. She stated that four months previously she had hen left eye-tooth pulled, after which she suffered violent pain for six weeks in her forehead, followed by pain and swelling in the left infraorbital region. She did not notice any imperfection of sight until.the end of March, when she perceived a mist in front of her left eye, which had 